\title{
Sítios não Controlados e sua utilização em pesquisas de geofísica forense com GPR: Uma Revisão Sistemática da Literatura
}

Kimberly Coutinho Paes Leme de Castro', Luciano Soares da Cunha², ${ }^{2}$ Universidade de Brasília - UnB, ${ }^{2}$ Universidade de Brasília - UnB

Copyright 2021, SBGf - Sociedade Brasileira de Geofísica.

This paper was prepared for presentation during the $17^{\text {th }}$ International Congress of the Brazilian Geophysical Society held in Rio de Janeiro, Brazil, $16-19$ August 2021 .

Contents of this paper were reviewed by the Technical Committee of the $17^{\text {th }}$ International Congress of the Brazilian Geophysical Society and do not necessarily represent any position of

the SBGf, its officers or members. Electronic reproduction or storage of any part of this paper for commercial purposes without the written consent of the Brazilian Geophysical Society is prohibited.

\section{Abstract}

The geophysical method plays an important role in the non-destructive detection of buried or hidden targets. The non-invasive nature of geophysical methods is of particular value in forensic geophysics and can provide quick answers in criminal investigations. It is common to use ground penetration radar (GPR) which can be used for various forensic research that seeks to find unmarked graves in old cemeteries as an aid in police investigations. The systematic literature review is a bibliographic research technique that allows the identification, evaluation, and interpretation of a set of relevant studies within an area of knowledge, thus producing a synthesis of a defined theme. The question raised in this review was to know what are the characteristics of uncontrolled sites used in forensic geophysics, the data acquisition parameters in these places, and, consequently, where the research on the subject is going. From the analysis of the results, it is possible to see that the scientific research carried out on the subject is concentrated between the years 2007 and 2019. The most frequently investigated sites were cemeteries and the most frequently investigated targets were human bodies. A new scope for further analysis is the research on false-positive results which was discussed in only $5.8 \%$ of the articles reviewed.

A Revisão Sistemática de Literatura (RSL) é uma técnica de investigação científica com objetivo de reunir, avaliar criticamente e conduzir uma síntese dos resultados de múltiplos estudos primários (BENEFIELD, 2003).

Para execução desta revisão foi utilizado o software StArt (Lapes/UFScar) e os artigos científicos foram pesquisados em três bases de dados: Sciense Direct, Web of Science e Scopus com as buscas estruturadas na seguinte string ((("GPR" OR "ground penetrating radar") AND "forensic" AND ("burial" OR "burials" OR "buried") AND ("graves" OR "grave"))).

Foram retornados 17 artigos sobre sítios não controlados utilizando o GPR com publicações ocorreram entre os anos de 2007 e 2019.

O sítio investigado com maior frequência foi cemitério em $41,1 \%$ dos artigos revisados, além de investigações de sepulturas clandestinas e valas comuns $(17,6 \%)$. Os tipos de solos que mais estiveram presentes foram os solos argilosos encontrados em $41,1 \%$ dos artigos e arenosos que apareceram em $35,2 \%$ dos artigos.

Sejam em sepultamentos de crimes recentes, sejam em cemitérios onde os corpos já estão enterrados há anos os alvos buscados em sua grande maioria eram corpos humanos, presentes em $94,1 \%$ dos artigos e em $5,9 \%$ dos artigos o alvo buscado foi o túmulo de um cão enterrado.

As frequências centrais utilizadas nos levantamentos com o GPR variaram de $100 \mathrm{MHz}$ a $500 \mathrm{MHz}$. As frequências mais utilizadas foram as de $500 \mathrm{MHz}$ (p.ex., BARONE \& DI MAGGIO, 2019) empregada em 35,2\% das investigações de alvo, a de $400 \mathrm{MHz}$ (p.ex., ABATE et al., 2019) e a de $250 \mathrm{MHz}$ (p.ex., WISNIEWSKI et al., 2019) ambas usadas em $23,5 \%$ dos artigos. A frequência de $500 \mathrm{MHz}$ apareceu mais frequentemente associada a solos arenosos, a de $400 \mathrm{MHz}$ esteve mais associada a solos argiloso enquanto a frequência de $250 \mathrm{MHz}$ apareceu associada a solos arenosos e argilosos.

Em 53\% dos artigos o GPR foi utilizado associado a outras técnicas complementares que foram bem variadas e incluíam: condutividade e suscetibilidade magnética (WISNIEWSKI et al., 2019), detector de metais (p.ex., RUFFELL et al., 2009a), fotografia aérea (p.ex., MCCABE et al., 2009a), eletrorresistividade (p.ex., PRINGLE et 
al., 2014), varredura a laser terrestre (p.ex., STEWART et al., 2016), cães de recuperação de vítimas (RUFFELL et al., 2009b), entre outros, enquanto nos outros $47 \%$ dos artigos foi utilizado apenas o método GPR para as análises de alvo.

Por meio da análise dos resultados desta RSL é possível concluir que as pesquisas em sítios não controlados visam prioritariamente evidenciar a importância da geofísica forense na detecção não destrutiva de alvos enterrados ou ocultos fornecendo respostas rápidas em investigações. Enquanto as pesquisas em sítios não controlados objetivam entender as limitações e aplicabilidade do GPR as pesquisas em sítios não controlados buscam apresentar resultados de pesquisa, documentar investigações, determinar a localização de sepulturas não marcadas, além de localizar restos mortais humanos. Resultados de uma análise GPR devem ser interpretados por indivíduos com experiência. Um novo escopo a ser explorado nas pesquisas de sítios não controlados é a análise de resultados falsos positivos e falsos negativos, algo muito pouco debatido nos artigos revisados.

Referências:

ABATE, D.; STURDY COLLS, C.; MOYSSI, N.; et al. Optimizing search strategies in mass grave location through the combination of digital technologies. Forensic Science International: Synergy, v. 1, p. 95-107, 2019. AZIZ, Azie S.; STEWART, Robert R.; GREEN, Susan L.; et al. Locating and characterizing burials using 3D ground-penetrating radar (GPR) and terrestrial laser scanning (TLS) at the historic Mueschke Cemetery, Houston, Texas. Journal of Archaeological Science: Reports, v. 8, p. 392-405, 2016.

BARONE, P.M.; SWANGER, K.J.; STANLEY-PRICE, N.; et al. Finding graves in a cemetery: Preliminary forensic GPR investigations in the Non-Catholic Cemetery in Rome (Italy). Measurement, v. 80, p. 53-57, 2016. BARONE, Pier Matteo ; DI MAGGIO, Rosa Maria. Forensic geophysics: ground penetrating radar (GPR) techniques and missing persons investigations. Forensic Sciences Research, v. 4, n. 4, p. 337-340, 2019. BENEFIELD, L. E. Implementing Evidence-Based Practice in Home Care. Home Healthcare Nurse: The Journal for the Home Care and Hospice Professional, [s. I.], v. 21, n. 12, p. 804-809, 2003. Disponível em: <https://pubmed.ncbi.nlm.nih.gov/14665967/>. Acesso em: 12 jun. 2021.

BILLINGER, Michael S. Utilizing Ground Penetrating Radar for the Location of a Potential Human Burial under Concrete. Canadian Society of Forensic Science Journal, v. 42, n. 3, p. 200-209, 2009.

BÜYÜKSARAÇ, Aydın; YALÇINER, Cahit Çağlar; EKINCI, Yunus Levent; et al. Geophysical investigations at Agadere Cemetery, Gallipoli Peninsular, NW Turkey. Australian Journal of Forensic Sciences, v. 46, n. 1, p. 111-123, 2013.

DAMIATA, Brian N.; STEINBERG, John M.; BOLENDER, Douglas J.; et al. Subsurface imaging a Viking-Age churchyard using GPR with TDR: Direct comparison to the archaeological record from an excavated site in northern Iceland. Journal of Archaeological Science: Reports, v. 12, p. 244-256, 2017.

DOOLITTLE, James A. ; BELLANTONI, Nicholas F. The search for graves with ground-penetrating radar in Connecticut. Journal of Archaeological Science, v. 37, n. 5, p. 941-949, 2010.

FERNÁNDEZ-ÁLVAREZ, José-Paulino; RUBIO-MELENDI, David; MARTíNEZ-VELASCO, Antxoka; et al. Discovery of a mass grave from the Spanish Civil War using Ground Penetrating Radar and forensic archaeology. Forensic Science International, v. 267, p. e10-e17, 2016. 
FIEDLER, Sabine; ILLICH, Bernhard; BERGER, Jochen; et al. The effectiveness of ground-penetrating radar surveys in the location of unmarked burial sites in modern cemeteries. Journal of Applied Geophysics, v. 68, n. 3, p. 380-385, 2009.

FORBES, Shari L.; HULSMAN, Steve ; DOLDERMAN, Mike. Locating Buried Canine Remains Using Ground Penetrating Radar. Canadian Society of Forensic Science Journal, v. 46, n. 1, p. 51-58, 2013.

HANSEN, James D.; PRINGLE, Jamie K. ; GOODWIN, Jon. GPR and bulk ground resistivity surveys in graveyards: Locating unmarked burials in contrasting soil types. Forensic Science International, v. 237, p. e14e29, 2014. Disponível em: <https://pubmed.ncbi.nlm.nih.gov/24559798/>. Acesso em: 12 Jun. 2021.

RUBIO-MELENDI, David; GONZALEZ-QUIRÓS, Andrés; ROBERTS, Daniel; et al. GPR and ERT detection and characterization of a mass burial, Spanish Civil War, Northern Spain. Forensic Science International, v. 287, p. e1-e9, 2018.

RUFFELL, Alastair; MCCABE, Alan; DONNELLY, Colm; et al. Location and Assessment of an Historic (150-160 Years Old) Mass Grave Using Geographic and Ground Penetrating Radar Investigation, NW Ireland. Journal of Forensic Sciences, v. 54, n. 2, p. 382-394, 2009a.

RUFFELL, Alastair; DONNELLY, Colm; CARVER, Naomi; et al. Suspect burial excavation procedure: A cautionary tale. Forensic Science International, v. 183, n. 1-3, p. e11-e16, 2009b.

SCHULTZ, John J. Using Ground-Penetrating Radar to Locate Clandestine Graves of Homicide Victims. Homicide Studies, v. 11, n. 1, p. 15-29, 2007.

StArt - LaPES - Laboratório de Pesquisa em Engenharia de Software. Software State of the Art through Systematic Review. Versão 3.4 Beta Ufscar.br. Disponível em: <http://lapes.dc.ufscar.br/tools/start_tool>. Acesso em: 26 Out. 2020.

WISNIEWSKI, Kristopher; COOPER, Nicholas; HEATON, Vivienne; et al. The Search for "Fred": An Unusual Vertical Burial Case. J Forensic Sci, 2019. 